\title{
Sinking loss should be taken into account while studying the dynamics of Microcystis under light-availability control
}

Yingying Huang ${ }^{\mathrm{a}}$, Chen Xuechu*1a,b ${ }^{\text {, }}$, Shengbing $\mathrm{He}^{\mathrm{b}}$, Yang An ${ }^{\mathrm{b}}$, Xiaojuan $\mathrm{Yu}^{\mathrm{b}}$, Xin Peng ${ }^{\mathrm{c}}$

${ }^{\text {a }}$ School of Ecology and Environmental Science, East China Normal University, Dong Chuan Road 500, Shanghai, 200241, P. R. China.

${ }^{\mathrm{b}}$ School of Environmental Science and Engineering, Shanghai Jiao Tong University, Dong Chuan Road 800, Shanghai, 200240, P. R. China.

${ }^{\mathrm{c}}$ Zhejiang Mariculture Research Institute, 6-1 Hetongqiao, Wenzhou, Zhejiang, 325005, P. R. China

\begin{abstract}
In-situ light-availability control is commonly used to suppress Microcystis blooms in nutrient-rich water resources. It has been suggested that the reduction of column cyanobacterial biomass could mostly be attributed to the inhibition of photosynthesis. However, sinking loss may be another factor influencing the column cyanobacterial biomass. To further investigate the mechanism of this reduction, a mixing-static water column experimental apparatus was designed to simulate the reduction of Microcystis biomass under light-availability control. Under light-shading plus mixing, the reduction of Microcystis in the water column was attributed to both intrinsic biomass loss and sinking loss.

Comparatively, under light-shading without mixing, the Microcystis accumulated in surface water, maintaining a continuously increase of intrinsic biomass. Meanwhile, the sinking loss increased as the water column became static, even exceeding the increase of intrinsic biomass, suggesting that sinking loss was the main mechanism for the reduction under light-shading. Further investigation indicated that both intrinsic growth rate and sinking loss rate varied in response to available light. Accordingly, a hypothesis is represented that the loss of column biomass and the shift in dominant species under light-availability control are primarily attributed to the combined effects of intrinsic biomass change and sinking loss, which both respond to available light.
\end{abstract}

Keywords

Microcystis bloom, Reduction, Light-shading, Mixing, Sinking loss

${ }_{1} *$ Corresponding author Tel: +86-21-54341137 FAX: +86-21-54341137 E-mail address: xcchen@des.ecnu.edu.cn 


\section{Introduction}

The cyanobacterium Microcystis is a widely distributed organism, and it dominates the phytoplankton community in many nutrient-rich drink water resources. Microcystis produces gas vesicles, which causes it to exhibit a lower density than water. When the water column is stable, the buoyant Microcystis floats upwards, forming dense surface blooms [1, 2], and enhances its own access to light while shading other algae. As a result, considerable water-quality problems occur due to bloom formation and the possible production of toxins and off-flavors. In order to ensure the safety of local water supply systems, various pre-treatment measures have been investigated including dissolved air flotation [3], chlorine or permanganate oxidation [4, 5], ultrasonic radiation [6] and ozonation [7]. However, these measures may be unacceptable because of high operating cost or release of some toxic substances such as microcystines (MCs). In situ cyanobacterial control is an alternative idea for the removal of cyanobacteria in water source area but not in water treatment plant. Currently, a simple physical measure termed in-situ "light-availability control" had been suggested [8-11]. There are two means of light-availability control: "artificial mixing" and "surface light-shading". Artificial mixing entrains surface Microcystis into the aphotic layer with prolonged darkness, until the cyanobacteria return to the euphotic layer. This inhibits Microcystis growth by reducing the available light $[10,12]$. Surface light-shading entails building a light-shading zone around the water intake location, forcing high-cyanobacterial water to stay in a dark area for a certain period, leading to a significant reduction of the cyanobacterial biomass $[8,9,11]$. Those previous studies attributed the reduction of biomass to the inhibition of photosynthesis in Microcystis. However, several batch tests suggested that Microcystis can adapt to and endure the stress of darkness, and the rate of reduction of the Microcystis population under light-limited conditions was relatively low $[13,14]$. Comparatively, field applications had confirmed that, in water treated with light-availability control, the Microcystis biomass in water column visibly decreased within a few days $[9,15]$. Such a contradiction between batch tests and field studies indicates that there may be another mechanism regulating Microcystis biomass in water column.

In normal conditions, the reported reduction of biomass by pre-treatment measures was actually the reduction of apparent biomass in overlying water. This value is a combination of the amounts due to sink loss and intrinsic biomass loss. Sinking loss of phytoplankton is a factor that influences phytoplankton biomass in water columns, especially when the mixing depth is low [16-18]. However, previous technical studies of light-availability control rarely measured the sinking loss of Microcystis 
directly, instead considering it as a small constant that would not significantly influence the biomass in the column. However, the contradiction mentioned above makes this assumption questionable.

In this study, batch tests were conducted with Microcystis aeruginosa and Scenedesmus obliquus to investigate the trends of biomass and specific density under different light incubation conditions. Meanwhile, a mixing-static water column experimental apparatus was designed to simulate the reduction of Microcystis biomass under light-availability control. The "light-shading" conditions were simulated within the apparatus by controlling the incident light and mixing process. During the experiment, the biomass in the overlying water and the sinking biomass at the bottom were measured. Therefore, the sinking loss and intrinsic biomass loss could be further distinguished, and these values were compared between different treatments to illustrate the mechanisms for the reduction of cyanobacterial biomass under light-availability control.

\section{Materials and Methods}

\subsection{Experimental design}

\subsubsection{Batch tests}

M. aeruginosa (No. FACHB469) and S. obliquus (No. FACHB416) were obtained from the Freshwater Algae Culture Collection of the Institute of Hydrobiology, Chinese Academy of Sciences, Wuhan, China. M. aeruginosa lacks colony organization when cultured under laboratory conditions. $S$. obliquus grows in four-celled colonies, known as coenobia. Before the experiment, M. aeruginosa and S. obliquus were cultured in BG-11 medium [19] until the exponential phase. Batch tests were conducted in 500-mL Pyrex bottles with $200 \mathrm{~mL}$ sterile BG-11 medium. M. aeruginosa and S. obliquus were inoculated, using the same inoculation cell concentration of $0.5 \times 10^{5}$ cells $\mathrm{mL}^{-1}$. The bottles were placed in a growth chamber. The light source was a white fluorescent light in a light:dark cycle of $14 \mathrm{~h}: 10$ $\mathrm{h}$ and at $25 \pm 1{ }^{\circ} \mathrm{C}$. Adjustments of shade cloth were used to obtain different incident illuminations, including 200 lx, 1000 lx and 5000 lx, and each treatment had two duplicates. Water temperature was controlled at $25 \pm 1{ }^{\circ} \mathrm{C}$ with a thermostat. Approximately 5 -ml samples were taken every 2 days for determination of chlorophyll a (Chl a) and specific density. The experiment lasted until the end of the exponential phase.

\subsubsection{Column experiments}

The simulation experiment was conducted in when Microcystis blooms frequently occurred in Taihu lake. Approximately $30 \mathrm{~L}$ of water with high Microcystis biomass was collected from the surface of 
Yangwan Bay, Taihu lake (31 $\left.31^{\prime} 58.88^{\prime \prime N}, 120^{\circ} 9^{\prime} 41.90 " \mathrm{E}\right)$ on August 1st, 2012, and immediately delivered to the lab (Minhang campus, Shanghai Jiaotong University). The water was diluted with lake water (collected from Siyuan Lake in the campus) to approximately $600 \mathrm{~L}$ and pre-cultured outside in a culture tank (1.2 $\mathrm{m}$ in height and $0.9 \mathrm{~m}$ in diameter). To maintain the activity of Microcystis and allow it to adapt to the new circumstances, $\mathrm{NaNO}_{3}$ and $\mathrm{KH}_{2} \mathrm{PO}_{4}$ were added to the water to ensure $\mathrm{TN} \geq 4 \mathrm{mg} \mathrm{L}-1$ and $\mathrm{TP} \geq 0.2 \mathrm{mg} \mathrm{L}^{-1}$, and aeration was conducted with an airflow rate of 120-150 mL minute ${ }^{-1}$. After 2 days of adaption, the aeration was stopped to facilitate the up-float of buoyant Microcystis. Then, approximately $30 \mathrm{~L}$ of high-cyanobacterial surface water was collected. The high-cyanobacterial water was diluted with lake water such that $\mathrm{Chl}$ a approached $200 \mu \mathrm{g} \mathrm{L^{-1 }}$. After that, the water was transferred to simulation experimental apparatuses in growth chamber reaching a height of $77 \mathrm{~cm}$ in each. $\mathrm{NaNO}_{3}$ and $\mathrm{KH}_{2} \mathrm{PO}_{4}$ were again added $\left(\mathrm{TN} \geq 4 \mathrm{mg} \mathrm{L}^{-1}, \mathrm{TP} \geq 0.2 \mathrm{mg} \mathrm{L}^{-1}\right)$.

A mixing-static water column experimental apparatus was designed to simulate the reduction of cyanobacterial biomass under light-availability control, as illustrated in Fig. 1. The experimental apparatus was a cylindrical column with a sedimentation chamber at the bottom. The whole column was $1 \mathrm{~m}$ in height and $20 \mathrm{~cm}$ in diameter, wrapped with black cloth. The sedimentation chamber was a honeycomb structure, approximately $7 \mathrm{~cm}$ in height and $8 \mathrm{~cm}$ in diameter. The light source was a white fluorescent light in a light:dark cycle of $14 \mathrm{~h}: 10 \mathrm{~h}$. Different light conditions were simulated by adjusting incident light with shade cloth. Mixing was simulated by an aerator with airflow rate of $8-10 \mathrm{~mL} \mathrm{~min}^{-1}$. The aerator was situated $10 \mathrm{~cm}$ above the sedimentation chamber, and preliminary experiments had confirmed that the trapped Microcystis in sedimentation chamber would not resuspend during mixing.

There were four treatments: light-shading (1000 lx) plus mixing, high-light (5000 lx) plus mixing, light shading (1000 lx) and control (5000 lx). Each treatment had two duplicates. Before sampling, the column water was well mixed for 30 seconds to make the biomass evenly distributed in the water column, with an airflow rate of 40-50 mL minute ${ }^{-1}$. After that, approximately $80-100 \mathrm{~mL}$ of column water was collected. At the same time, the sedimentation chamber was lifted slowly, and the water with sunken Microcystis was collected. Then, the sedimentation chamber was returned to the bottom of the column, and lake water was supplemented to maintain the water level. Collected water samples were taken for determination of $\mathrm{Chl}$ a. The experiment continued for 10 days.

To further address the reason for the succession of dominant species, water high in Microcystis was mixed with $S$. obliquus to simulate the competition of these two species under light-shading plus mixing. 
The experimental apparatus and sampling method was as same as that mentioned above, and the treatment had two duplicates.

\subsection{Analyses}

The algal biomass was determined by PHYTO-PAM, (WALZ, Germany, Phytowin v1.32). Based on the relative proportions of pigments present in phytoplankton species, PHYTO-PAM analysis estimates the relative biomasses of two algae [20], and the average specific growth rate was calculated accordingly. The specific density of algae was measured by the Percoll density gradient centrifugation method [21]. Percoll was mixed with growth medium in the proportion of 2.7 to 1 . Algal samples of approximately 2 $\mathrm{mL}$ were added to the mixture. The mixture was then centrifuged at $14000 \mathrm{rpm}$ for $30 \mathrm{~min}$. After that, a stable density gradient was formed, with the cells or colonies banded at their buoyant density during the centrifugation [22]. The specific density of each visible phytoplankton band was measured with a kerosene- $\mathrm{CCl}_{4}$ column [23] of a known density gradient.

To better understanding the mechanisms behind the cyanobacterial biomass reduction, the raw data (the Chla in overly water and sedimentation chamber) was further processed, and some specific terms were used as following: I. the column biomass (Standing stock of biomass). It was calculated by: multiplying the measured $\mathrm{Chl}$ a in overlying water by the mixing depth $(70 \mathrm{~cm})$; II. the daily sinking biomass. It was calculated by: multiplying the measured $\mathrm{Chl}$ a in sedimentation chamber by the height of sedimentation chamber $(7 \mathrm{~cm})$. In order to calculate the biomass balance, the daily sinking biomass was represented as a negative value; III. the intrinsic biomass. It was calculated by: subtracting the daily sinking biomass from the column biomass in overlying water; IV. the overall column biomass change. It was calculated by: subtracting the initial column biomass in overlying water from the column biomass on the $10^{\text {th }}$ day; V. the overall sinking loss. It was calculated by: summing the daily sinking biomass during the experiment; and VI. the overall intrinsic biomass change (the overall growth/death biomass). It was calculated by: subtracting the value of the overall sinking loss from the value of the overall column biomass change.

\section{Results}

\subsection{Growth of two species under different light incubation conditions}

Fig. 2 shows the growth of M. aeruginosa and S. obliquus under different light incubation conditions. At an incident illumination of 200 lx, the growth of M. aeruginosa was limited, and the specific density continuously decreasing from 1.018 to 1.015 . In contrast, an obvious exponential increase trend of $M$. 
aeruginosa was maintained at an incident illumination of $5000 \mathrm{~lx}\left(\mu=0.40, \mathrm{r}^{2}=0.973\right)$ and $1000 \mathrm{~lx}$ $\left(\mu=0.22, r^{2}=0.961\right)$, and a continuous increasing trend of specific density was observed. Increasing trends of S. obliquus biomass were maintained in all treatments. At incident illumination of $200 \mathrm{~lx}$, unlike M. aeruginosa, S. obliquus appeared to adapt to such low light-availability of 200 lx, and the Chl a exponentially increased from $69.2 \mu \mathrm{g} \mathrm{L}^{-1}$ to $309.8 \mu \mathrm{g} \mathrm{L}^{-1}$ during 10 days' incubation $(\mu=0.16$, $\left.\mathrm{r}^{2}=0.996\right)$. Meanwhile, the specific density of $S$. obliquus increased under all the light treatments; and a maximal increase of specific density was maintained at the incident illumination of $5000 \mathrm{~lx}$.

\subsection{Change of column biomass and sinking biomass under light-availability control}

Fig. 3a shows the change in column biomass under light-availability control. During this experiment, rapid reductions in Microcystis were observed under three types of treatments. In light-shading plus mixing column, the column biomass decreased from $169.4 \mathrm{mg} \mathrm{Chl} \mathrm{a} \mathrm{m}^{-2}$ to $13.5 \mathrm{mg} \mathrm{Chl} \mathrm{a} \mathrm{m}^{-2}$, with the highest reduction efficiency of $92 \%$. In the light-shading column, a similar decreasing trend was observed. However, the reduction efficiency was lower under this treatment by approximately $85 \%$. In the control, the column biomass decreased within the first two days and then varied slightly, remaining higher than that in the other columns. These results verified that the light-availability control could significantly reduce Microcystis in overlying water, as reported previously.

Fig. $3 \mathrm{~b}$ shows the change of daily sinking biomass under light-availability control. Interestingly, a continuous sinking loss of Microcystis was observed in all treatments. In column with treatment of light-shading plus mixing, the daily sinking biomass was relatively low, ranging from $-3.7 \mathrm{mg} \mathrm{Chl} \mathrm{a} \mathrm{m}{ }^{-2}$

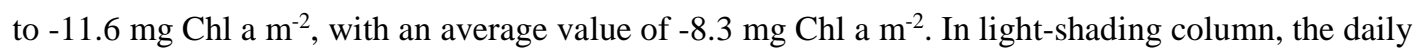

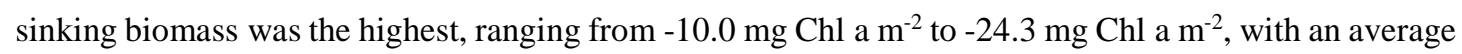
value of $-16.5 \mathrm{mg} \mathrm{Chl} \mathrm{a} \mathrm{m}{ }^{-2}$. In control column, the daily sinking biomass ranged from $-10.7 \mathrm{mg} \mathrm{Chl}$ a

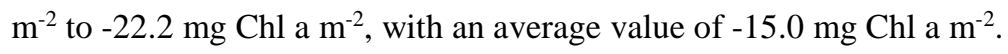

\subsection{Cyanobacterial biomass balance}

Although it was clear that the Microcystis in overlying water would be reduced by light-availability control, the mechanism underlying this phenomenon should be further investigated, considering that sinking loss influences this reduction. Therefore, the intrinsic biomass was calculated to illustrate the net change of biomass. As shown in Fig. 4a, in the light-shading plus mixing column, a continuous decrease of intrinsic biomass was observed. In contrast, in the high-light mixing column and the light-shading column, no obvious decrease in intrinsic biomass was observed. These results were interesting, 
considering that a continuous decrease of column biomass was observed at the same time.

To further illuminate the cyanobacterial biomass balance under light-availability control, the overall column biomass change, overall sinking loss and overall intrinsic biomass change were calculated (Fig 4b). This result indicated a same net loss of column biomass for these three treatments. However, the main reasons for the reduction were different. In the light-shading plus mixing column, the growth of Microcystis was strongly inhibited, and both sinking loss and intrinsic biomass change were responsible for the reduction. However, in the high-light column plus mixing and the light-shading column, sinking loss alone was responsible for the reduction. Meanwhile, the growth of Microcystis continued, and the increase of overall intrinsic biomass could partly compensate for the overall sinking loss.

\subsection{Competition between Microcystis and S. obliquus under light-availability control}

As shown in Fig. 5, rapid reductions in the column biomass of both Microcystis and S. obliquus were observed during this experiment. The column biomass of Microcystis decreased from $93.1 \mathrm{mg} \mathrm{Chl} \mathrm{a} \mathrm{m}{ }^{-2}$ to13.6 mg Chl a m${ }^{-2}$ within 7 days. Comparatively, S. obliquus decreased more slowly, from $90.8 \mathrm{mg}$

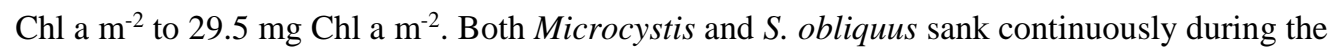
experiment, while the sinking biomass of Microcystis decreased gradually with treatment time. After 7 days, the overall sinking loss of Microcystis was $-54.4 \mathrm{mg} \mathrm{Chl} \mathrm{a} \mathrm{m}{ }^{-2}$, while the value of S. obliquus was $-73.7 \mathrm{mg} \mathrm{Chl} \mathrm{a} \mathrm{m}^{-2}$. Growth of $S$. obliquus maintained under such conditions, with a net increase of

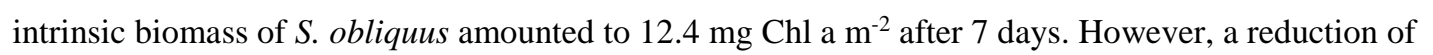
intrinsic biomass of Microcystis was observed, with a net loss of intrinsic biomass of $25.1 \mathrm{mg} \mathrm{Chl} \mathrm{a} \mathrm{m}^{-2}$ after 7 days. This result suggested the main advantage for S. obliquus was in terms of survivality and continuous growth under conditions of low availability light, whereas Microcystis could not.

\section{Discussion}

\subsection{Reasons for reduction of Microcystis under light-availability control}

The advantage of Microcystis is that it can produce gas vesicles, which provide buoyancy and allow access to well-illuminated surface waters, and it can regulate its buoyancy according to available light $[24,25]$. Previous studies have shown that "light-shading" can reduce cyanobacterial biomass and it induces surface accumulation of Microcystis $[9,26]$. Interestingly, the results of the simulation experiment suggested that sinking loss, rather than intrinsic biomass loss, was the main mechanism for this reduction. On the contrary, the intrinsic biomass of Microcystis increased upon such treatment. It should be noted that, according to field applications, after surface water was shaded with shading cloth, 
approximately 3-5\% incident light could penetrate into the surface water. To simulate light-shading, during our experiment, the incident light was maintained at 1000 lx. Therefore, because Microcystis accumulated in surface water under these conditions, it could absorb surface light to maintain the growth of intrinsic biomass. It was probably the main reason for the increase of intrinsic biomass. Previous studies also showed that the reduction rate of cyanobacterial biomass was higher under light-shading plus mixing than under light-shading condition [8]. The result of our simulation experiment confirmed this phenomenon, considering an obvious intrinsic biomass loss of Microcystis under conditions of light-shading plus mixing. Meanwhile, the result further suggested that the sinking loss should not be ignored, as it accounted for a large amount of the biomass loss in the water column. The sinking loss might be a common phenomenon regulating Microcystis dynamics in overlying water, regardless of whether the cyanobacteria was under light-limited conditions or not.

Moreover, it was observed that light-availability control would lead to a significant decrease of cyanobacterial biomass and changes the dominant species in the water column from buoyant cyanobacteria to green algae and diatoms $[27,28]$. However, few studies had distinguished the contributions of sinking loss and intrinsic biomass changes in this competition. The result of simulation experiment indicated that succession was driven by the combined effects of intrinsic biomass change and sinking loss. Such deduction can be proved by the results of the batch test. At incident illumination of 200 lx, the growth of Microcystis was limited; however, S. obliquus appeared to adapt to low light availability, showing a significant increase of biomass during 10 days of incubation. Accordingly, in column experiment, the column biomass of S. obliquus remained higher than Microcystis, mainly due to the net increase of intrinsic biomass, although it sank faster than Microcystis.

\subsection{Influence of available light on intrinsic biomass change and sinking loss}

Our experiments demonstrated that the intrinsic growth and sinking loss of Microcystis varied among different treatments. Such phenomena might be attributed to the influence of available light. For mixing, the available light could be estimated by integrating the light density over the depth, as follows [29]:

$$
I_{a}=\int_{0}^{z_{\text {mix }}} I_{\mathrm{s}} \mathrm{d} z=\frac{I_{0}}{K}\left(1-\mathrm{e}^{-k z_{\text {mix }}}\right)
$$

where $k$ is the light attenuation coefficient $\left(6.27 \mathrm{~m}^{-1}\right.$ for high-light plus mixing, $6.56 \mathrm{~m}^{-1}$ for light-shading plus mixing), $I_{o}(\mathrm{~lx})$ is the incident light, $\mathrm{z}_{\mathrm{mix}}$ is the mixing depth ( $0.7 \mathrm{~m}$ for both columns). For light-shading without mixing, the water was static; thus, most Microcystis accumulated in the surface 
water. The available light approached the incident light. Therefore, the available light in the two mixing columns could be calculated as $150.8 \mathrm{~lx}$ and $778.0 \mathrm{~lx}$, and the available light in the light-shading column and the control column was approximately 1000 lx and 5000 lx, respectively. On the other side, the intrinsic growth rate and sinking loss rate can be calculated according to formulation represented in previous studies [30]. Fig 6 summarizes the available light, intrinsic growth rate, and sinking loss rate within different treatments. These results indicated that the intrinsic growth rate increased with the increase of available light and the available light may be the key factor regulating intrinsic biomass change within different treatments. For instance, under conditions of light-shading plus mixing, the intrinsic growth rate was lowest and negative because the available light was as low as 150.8 lx, which would not support the growth of Microcystis. However, in the control column, the intrinsic growth rate was highest because the available light was as high as 5000 1x. The influence of available light was consistent with the results of the batch test. As suggested by the batch test, the growth of Microcystis was inhibited at incident illumination of 200 1x, whereas the highest Microcystis growth rate was observed at the incident illumination of $5000 \mathrm{~lx}$.

Fig. 6 also indicated that the available light influenced the sinking loss rate. The sinking loss rate was the lowest under the condition of light-shading plus mixing, with the lowest available light of 150.8 lx. The lower sinking loss rate under conditions of light-shading plus mixing might be related to changes in the specific density of Microcystis. According to the batch test results, at incident illumination of $200 \mathrm{~lx}$, the specific density continuously decreased from 1.018 to 1.015 , whereas at $10001 \mathrm{x}$ and 5000 lx, a continuous increase trend of specific density was observed. Due to the consumption of carbohydrates, the specific density of Microcystis tends to decrease in response to low-light conditions [24]. The decrease of specific density may negatively influence the sinking velocity, thus lowering the sinking loss rate.

\subsection{Hypothesis for interpreting Microcystis dynamics under light-availability control}

According to the results of this study, a hypothesis is represented that the loss of column biomass under light-availability control is primarily attributed to the combined effect of intrinsic biomass loss and sinking loss, which both respond to available light. Normally, Microcystis survives in the euphotic layer, where light is sufficient. The column biomass of Microcystis gradually reaches a steady state, with the sinking loss rate equal to the intrinsic growth rate. Under light-shading plus mixing or deep mixing, the Microcystis is entrained from the euphotic layer to aphotic layer. Therefore, the intrinsic 
growth rate becomes negative due to the lack of available light. For the sinking loss, although it decreases with the deeper mixing depth and lower sinking velocity, it can still accounted for a large amount of the biomass loss in the water column. In response to the combined effect of intrinsic biomass loss and sinking loss, the column biomass of Microcystis quickly decreases to an extremely low level, and other algae that can grow continuously under low light, such as S. obliquus, succeed in competition. Comparatively, by light-shading without mixing, Microcystis is not entrained to deep water. Instead, it floats upwards and accumulates in surface water, where it can absorb the remaining available light. Therefore, the intrinsic growth rate of Microcystis remains positive, although much lower than before. Meanwhile, the sinking loss rate increases as the water column becomes static. The column biomass will decrease continuously when sinking loss rate exceeds the intrinsic growth rate.

The above hypothesis emphasizes that the sinking loss should be taken into account while studying the dynamics of Microcystis under light-availability control. However, in field applications, the light regime is complicated, and there still exist other loss mechanisms, including grazing by zooplankton and infection by bacteria. These losses may also change in response to available light. Nevertheless, in field applications of artificial mixing, when Microcystis is entrained to deep water, where water pressure is very high, its sinking loss may obviously increase due to the breakdown of gas vesicles [31]. Therefore, further studies should be conducted to confirm this hypothesis and integrate it into existing hypothesis.

\section{Conclusion}

1) The light-availability control could significantly reduce biomass of Microcystis in overlying water. The treatment with light-shading plus mixing inhibited the growth of Microcystis strongly, and both sinking loss and intrinsic biomass loss were responsible for the reduction. Comparatively, during light-shading treatment, sinking loss alone was responsible for the reduction. The intrinsic biomass of Microcystis increased continuously.

2) During the treatment with light-shading plus mixing, the growth of Microcystis decreased more quickly than S. obliquus. The advantage for S. obliquus was in survivality and continuous growth under conditions of low available light, whereas Microcystis could not.

3) Both intrinsic biomass change and sinking loss varied in response to available light. The intrinsic growth rate increased with the increase of available light, and the sinking loss rate decreased to lowest under the condition of light-shading plus mixing. 


\section{Acknowledgments}

The present work was supported by the National Science Foundation of China under Grant No.41001361 and No.41471393, and the Shanghai Key Lab for Urban Ecological Processes and Eco-Restoration. We thank Professor Varenyam Achal from School of Ecology and Environmental Science, East China Normal University for improving this paper.

\section{References}

[1] E. Aparicio Medrano, R.E. Uittenbogaard, L.M. Dionisio Pires, B.J.H. van de Wiel, H.J.H. Clercx, Coupling hydrodynamics and buoyancy regulation in Microcystis aeruginosa for its vertical distribution in lakes, Ecol. Model. 248 (2013) 41-56.

[2] B.W. Ibelings, Changes in photosynthesis in response to combined irradiance and temperature stress in cyanobacterial surface waterblooms, J. Phycol. 32 (1996) 549-557.

[3] M.R. Teixeira, V. Sousa, M.J. Rosa, Investigating dissolved air flotation performance with cyanobacterial cells and filaments, Water Res. 44 (2010) 3337-3344.

[4] E. Rodríguez, M.E. Majado, J. Meriluoto, J.L. Acero, Oxidation of microcystins by permanganate: Reaction kinetics and implications for water treatment, Water Res. 41 (2007) 102-110.

[5] X.J. Zhang, C. Chen, J.Q. Ding, A. Hou, Y. Li, Z.B. Niu, X.Y. Su, Y.J. Xu, E.A. Laws, The 2007 water crisis in Wuxi, China: Analysis of the origin, J. Hazard. Mater. 182 (2010) 130-135.

[6] X. Wu, E.M. Joyce, T.J. Mason, Evaluation of the mechanisms of the effect of ultrasound on Microcystis aeruginosa at different ultrasonic frequencies, Water Res. 46 (2012) 2851-2858.

[7] Z. Wu, H. Shen, B. Ondruschka, Y. Zhang, W. Wang, D.H. Bremner, Removal of blue-green algae using the hybrid method of hydrodynamic cavitation and ozonation, J. Hazard. Mater. 235-236 (2012) $152-158$.

[8] X. Chen, S. He, Y. Huang, H. Kong, Y. Lin, C. Li, G. Zeng, Laboratory investigation of reducing two algae from eutrophic water treated with light-shading plus aeration, Chemosphere 76 (2009) 1303-1307.

[9] X.C. Chen, H.N. Kong, S.B. He, D.Y. Wu, C.J. Li, X.C. Huang, Reducing harmful algae in raw water by light-shading, Process. Biochem. 44 (2009) 357-360. 
[10] M.A. Imteaz, T. Asaeda, Artificial mixing of lake water by bubble plume and effects of bubbling operations on algal bloom, Water Res. 34 (2000) 1919-1929.

[11] J.F. Maestre-Valero, V. Martínez-Alvarez, E. Nicolas, Physical, chemical and microbiological effects of suspended shade cloth covers on stored water for irrigation, Agr. Water Manage. 118 (2013) 70-78.

[12] L. Liu, D. Liu, D.M. Johnson, Z. Yi, Y. Huang, Effects of vertical mixing on phytoplankton blooms in Xiangxi Bay of Three Gorges Reservoir: Implications for management, Water Res. 46 (2012) 2121-2130.

[13] E. Furusato, T. Asaeda, J. Manatunge, Tolerance for prolonged darkness of three phytoplankton species, Microcystis aeruginosa (Cyanophyceae), Scenedesmus quadricauda (Chlorophyceae), and Melosira ambigua (Bacillariophyceae), Hydrobiologia 527 (2004) 153-162.

[14] Z. Wu, L. Song, R. Li, Different tolerances and responses to low temperature and darkness between waterbloom forming cyanobacterium Microcystis and a green alga Scenedesmus, Hydrobiologia 596 (2008) 47-55.

[15] J. Huisman, J. Sharples, J.M. Stroom, P.M. Visser, W.E.A. Kardinaal, J.M.H. Verspagen, B. Sommeijer, Changes in turbulent mixing shift competition for light between phytoplankton species, Ecology 85 (2004) 2960-2970.

[16] S. Diehl, Phytoplankton, light, and nutrients in a gradient of mixing depths: Theory, Ecology 83 (2002) 386-398.

[17] J. Padisák, É. Soróczki-Pintér, Z. Rezner, Sinking properties of some phytoplankton shapes and the relation of form resistance to morphological diversity of plankton - An experimental study, Hydrobiologia 500 (2003) 243-257.

[18] A. Pannard, M. Bormans, Y. Lagadeuc, Short-term variability in physical forcing in temperate reservoirs: Effects on phytoplankton dynamics and sedimentary fluxes, Freshwater Biol 52 (2007) $12-27$.

[19] R. Rippka, J. Deruelles, J.B. Waterbury, M. Herdman, R.Y. Stanier, Generic assignments, strain histories and properties of pure cultures of cyanobacteria, J. Gen. Microbiol. 111 (1979) 1-61.

[20] H. Walz, Phytoplankton Analyzer PHYTO-PAM: System components and principles of operation, Heinz Walz GmbH, Effeltrich, Germany, 1999.

[21] H. Pertoft, Fractionation of cells and subcellular particles with Percoll, J. Biochem. Bioph. Meth. 
$44(2000) 1-30$.

[22] D.A. Wolff, The separation of cells and subcellular particles by colloidal silica density gradient centrifugation, in: D.M. Prescott (Ed.) Methods Cell Biology, Academic press, United States, 1975, pp. $85-104$.

[23] S.A. Condie, M. Bormans, The influence of density stratification on particle settling, dispersion and population growth, J. Theor. Biol. 187 (1997) 65-75.

[24] J. Kromkamp, A.E. Walsby, A computer model of buoyancy and vertical migration in cyanobacteria, J. Plankton. Res. 12 (1990) 161-183.

[25] B.B. Wallace, M.C. Bailey, D.P. Hamilton, Simulation of vertical position of buoyancy regulating Microcystis aeruginosa in a shallow eutrophic lake, Aquat. Sci. 62 (2000) 320-333.

[26] V. Martínez-Alvarez, J.F. Maestre-Valero, Comparative Analysis of on-Farm Reservoir Management Techniques and Their Effect on Filtering Requirements for Irrigation, Water. Resour. Manag. 29 (2014) 1155-1167.

[27] M.A. Burford, M.J. O'Donohue, A comparison of phytoplankton community assemblages in artificially and naturally mixed subtropical water reservoirs, Freshwater Biol. 51 (2006) 973-982. [28] E. Jungo, P.M. Visser, J. Stroom, L.R. Mur, Artificial mixing to reduce growth of the blue-green alga Microcystis in Lake Nieuwe Meer, Amsterdam: An evaluation of 7 years of experience, Water Sci. Technol.: Water Supply 1 (2001) 17-23.

[29] K.E. Havens, E.J. Phlips, M.F. Cichra, B.L. Li, Light availability as a possible regulator of cyanobacteria species composition in a shallow subtropical lake, Freshwater Biol. 39 (1998) 547-556. [30] C. Reynolds, Chapter 2: entrainment and distribution in the pelagic, in: The Ecology of Phytoplankton, Cambridge University Press, Cambridge, 2006, pp. 38-92.

[31] D.I. Bright, A.E. Walsby, The relationship between critical pressure and width of gas vesicles in isolates of Planktothrix rubescens from Lake Zurich, Microbiology 145 (1999) 2769-2775. 


\section{Figure captions}

Fig. 1. Diagram of the mixing-static water column experimental apparatus

Fig. 2. The growth of M. aeruginosa (a) and S. obliquus (b) under different light incubation conditions

Fig. 3. Change of column biomass (a) and sinking biomass (b) under light-availability control

Fig. 4 Change of intrinsic biomass under light-availability control (a) and the overall column biomass, sinking biomass and intrinsic biomass (b)

Fig.5 Change of column biomass and sinking biomass of Microcystis and S. obliquus under light-shading plus mixing

Fig.6 The available light, intrinsic growth rate, and sinking loss rate within different treatments 
Figure 1

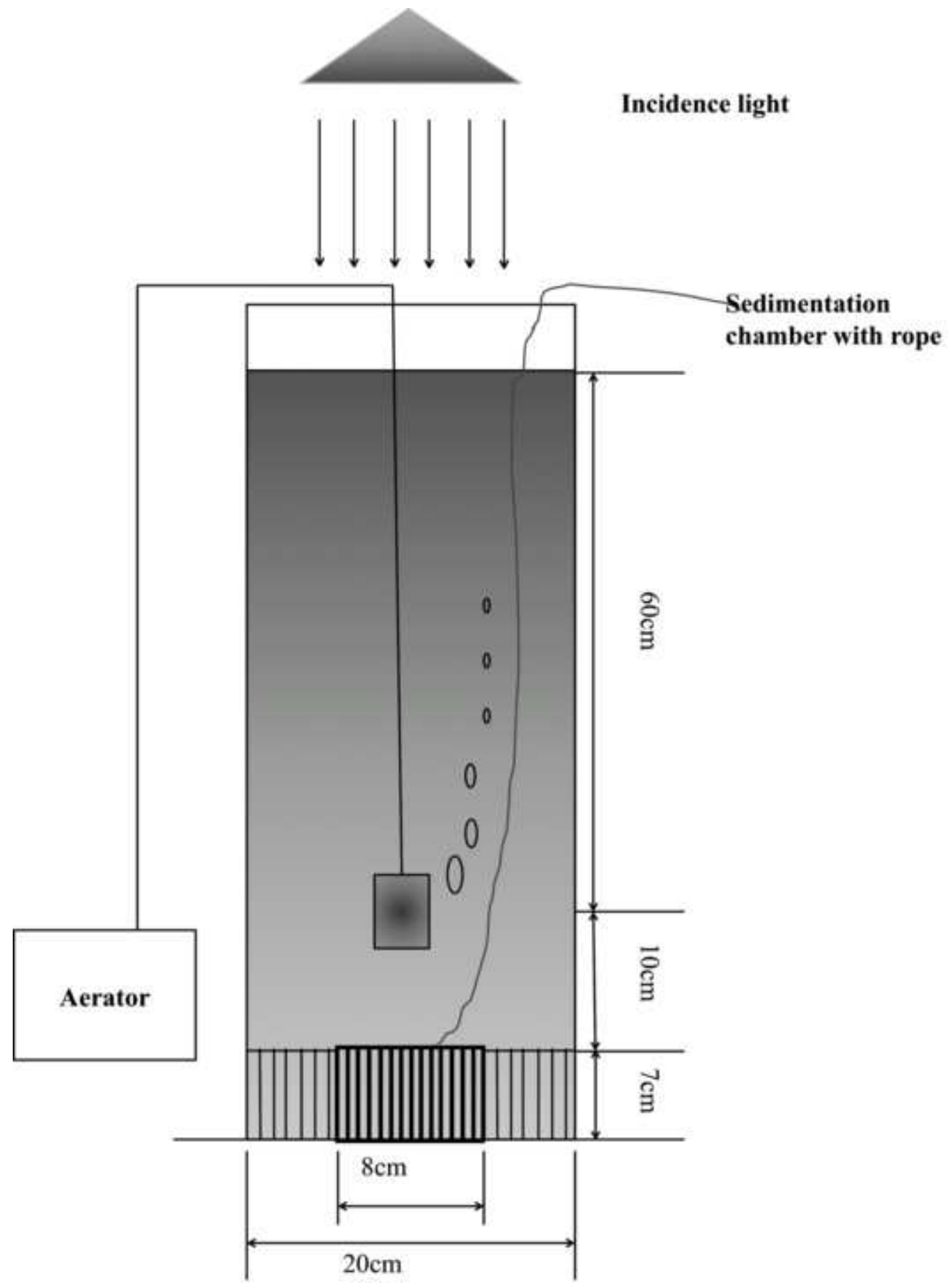

气ิ

章

$\breve{\Xi}$

\section{Aerator}

edimentation chamber with rope

\section{Incidence light}

(n)

Sedime 


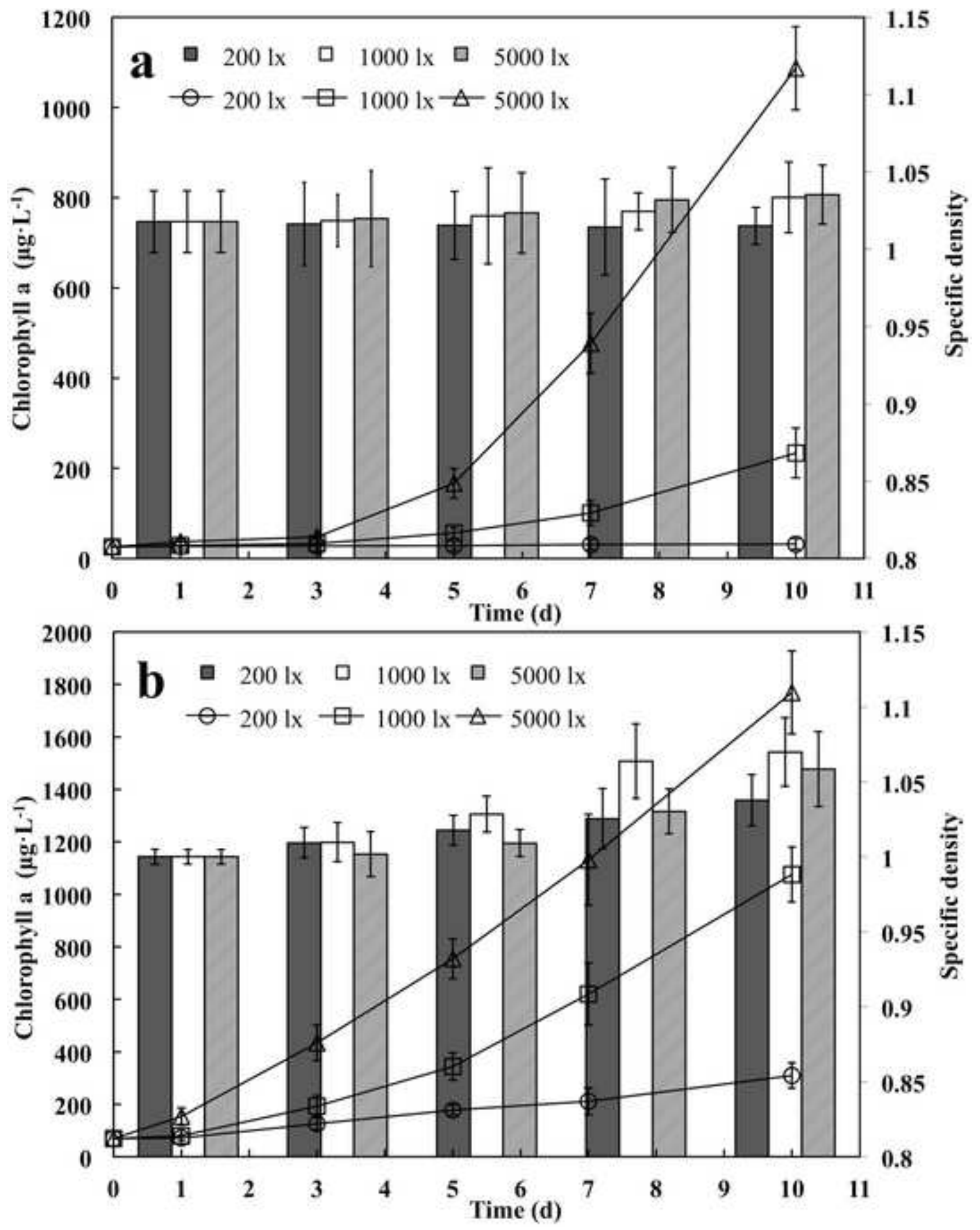



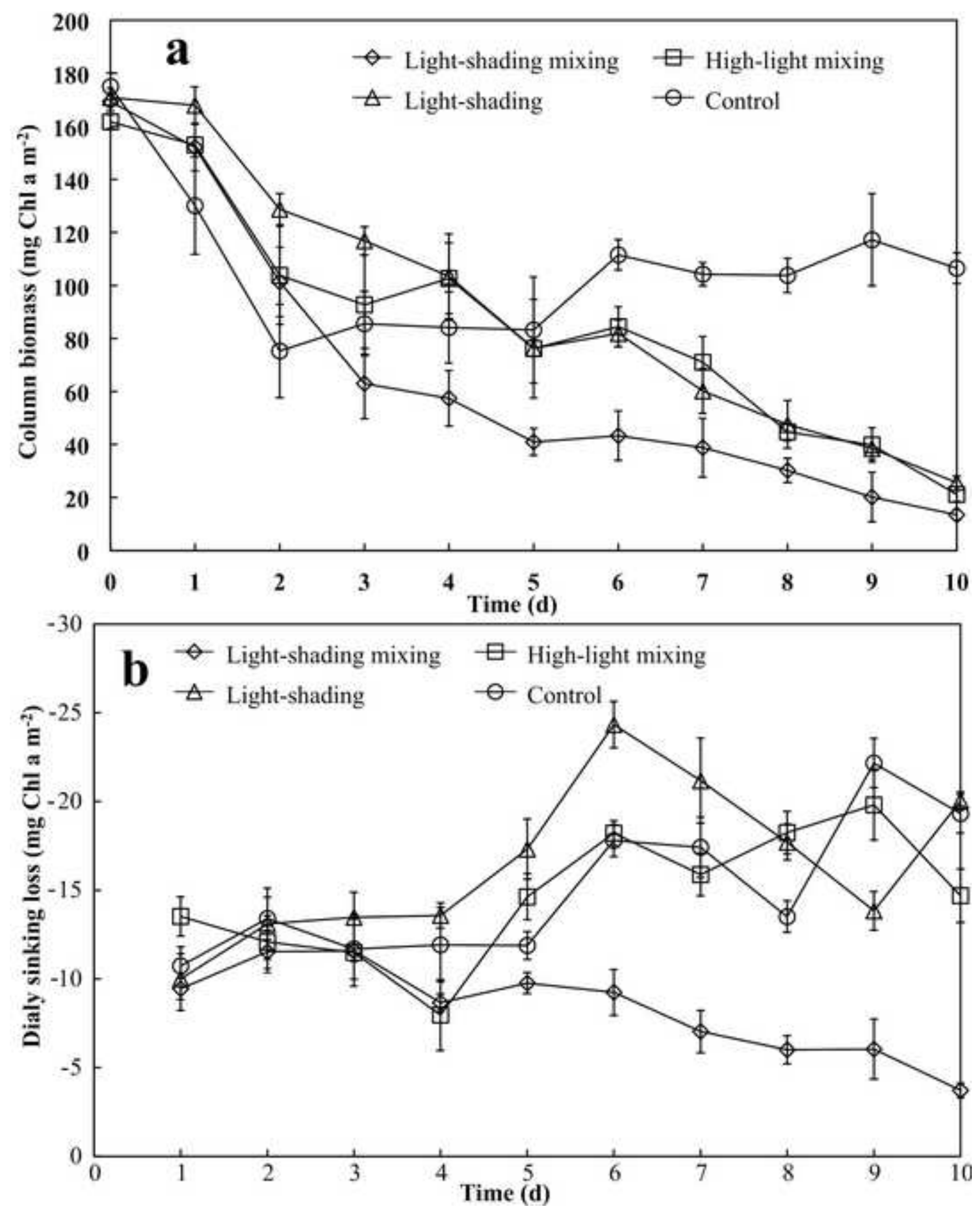

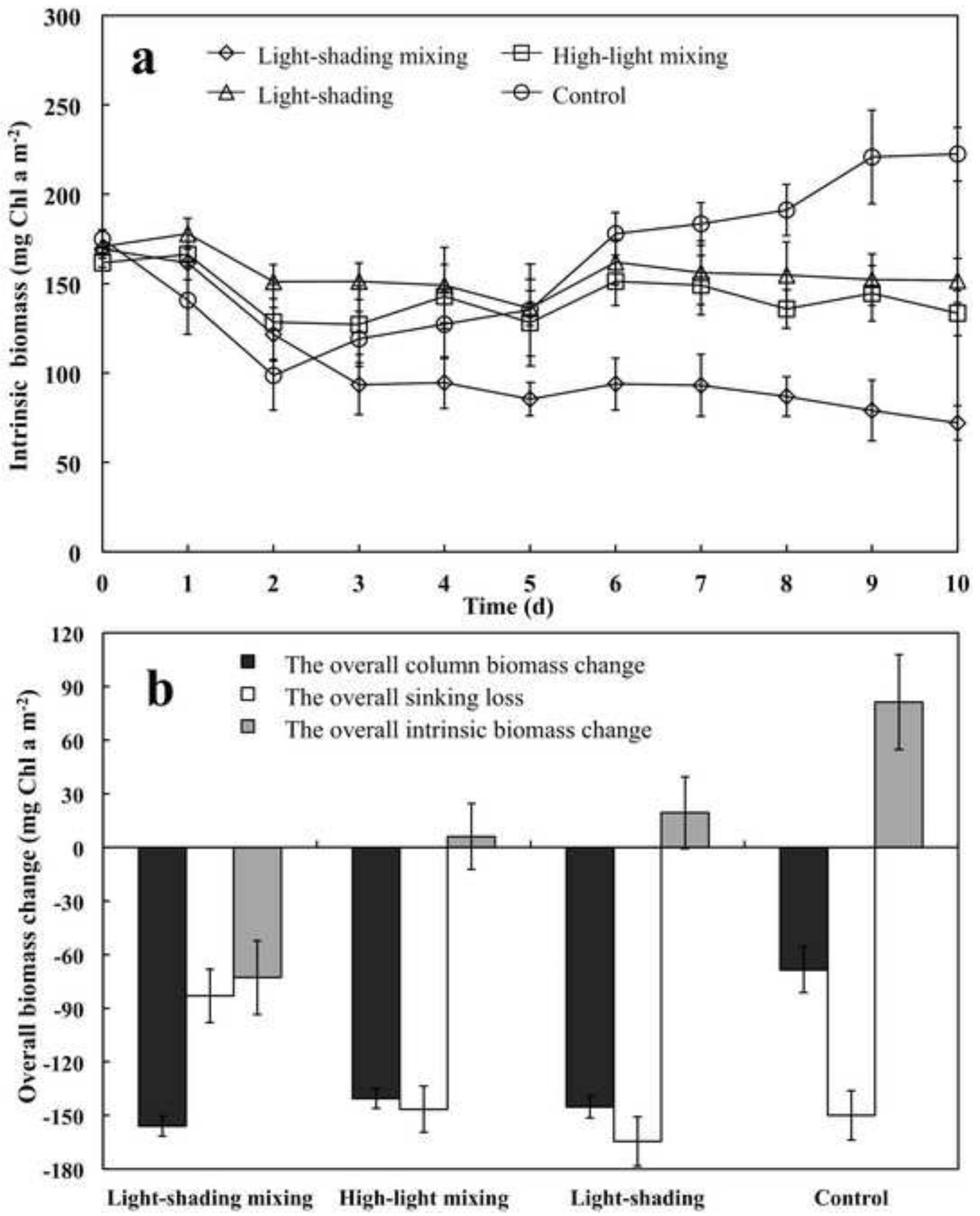


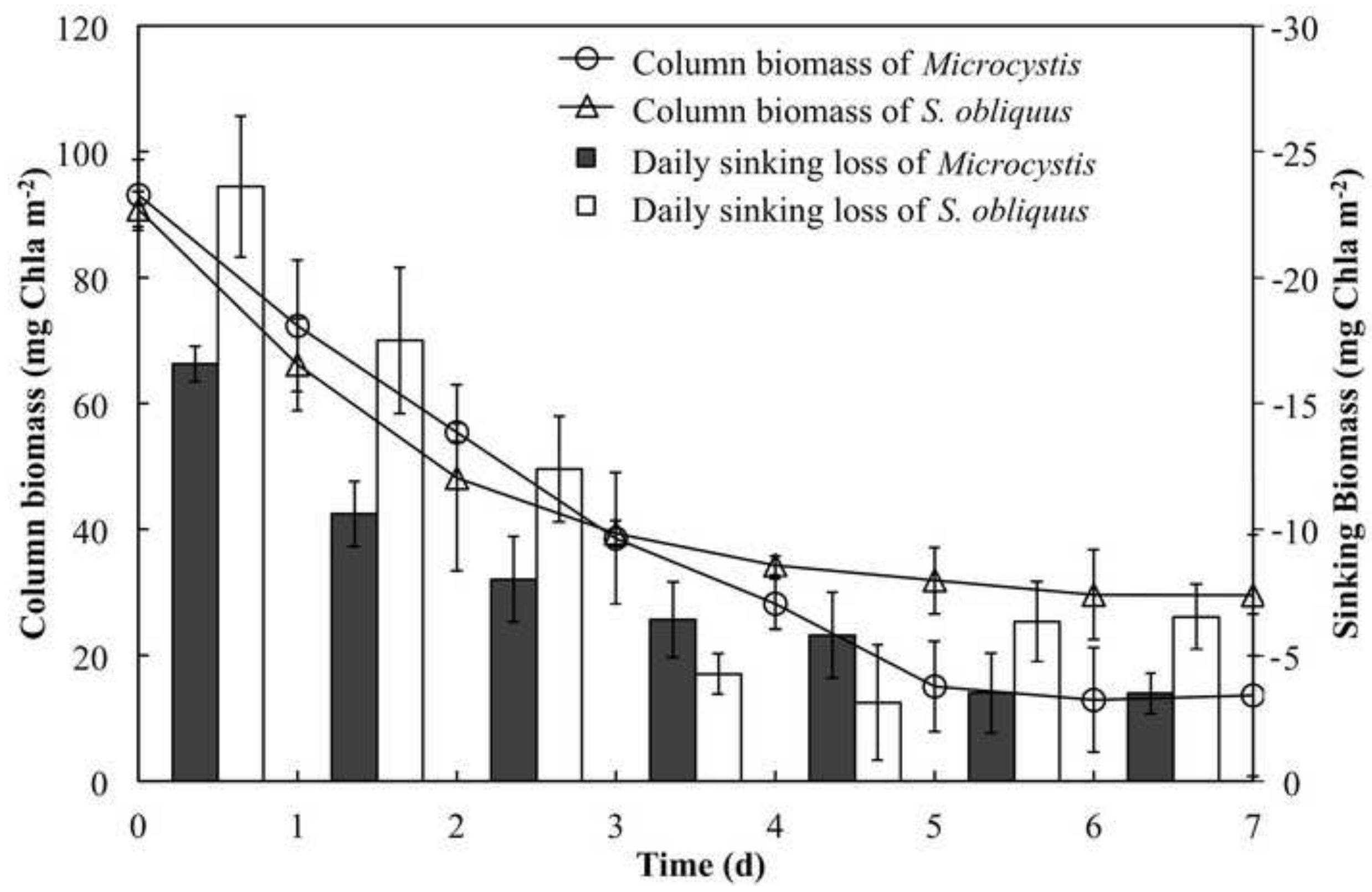




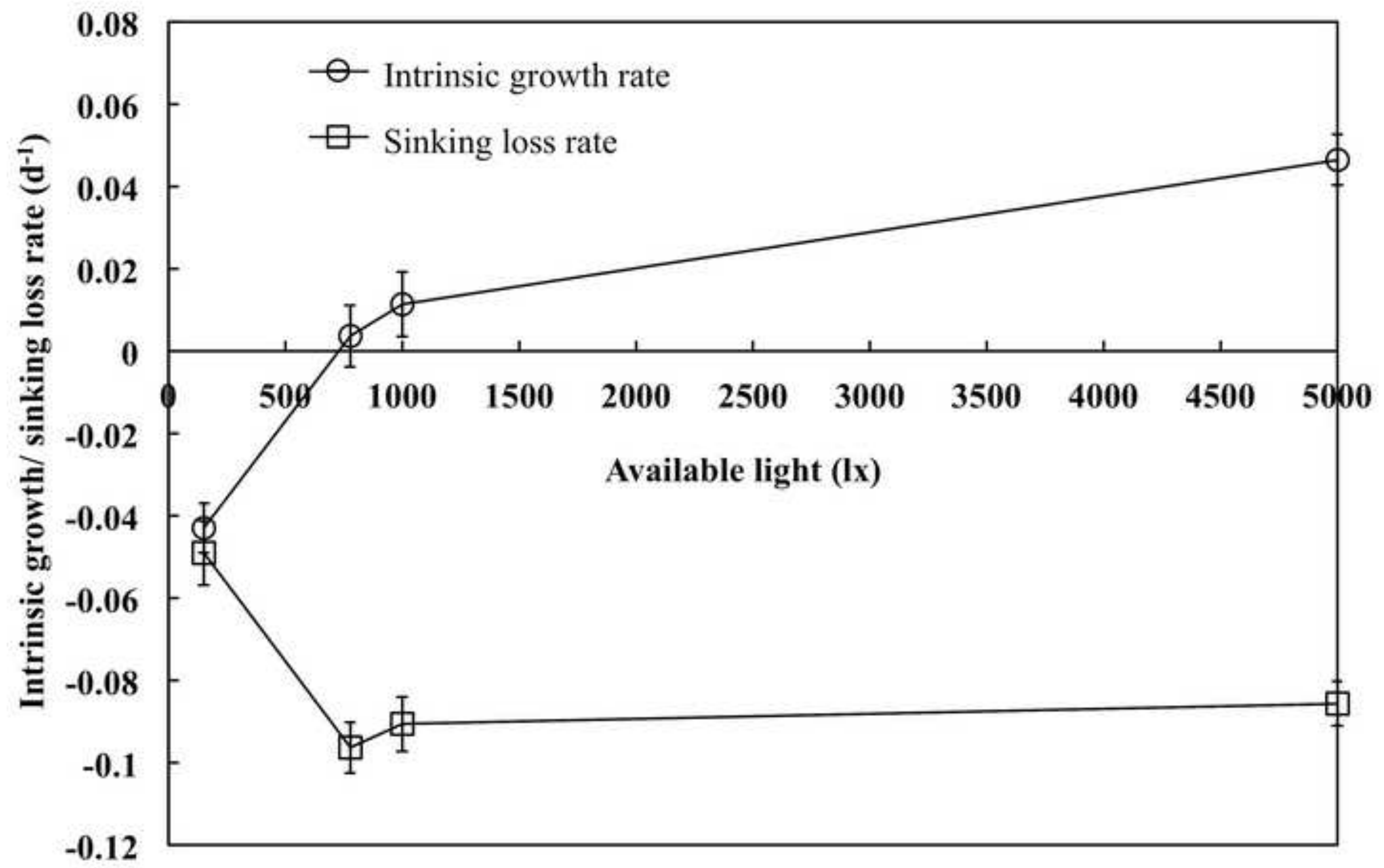



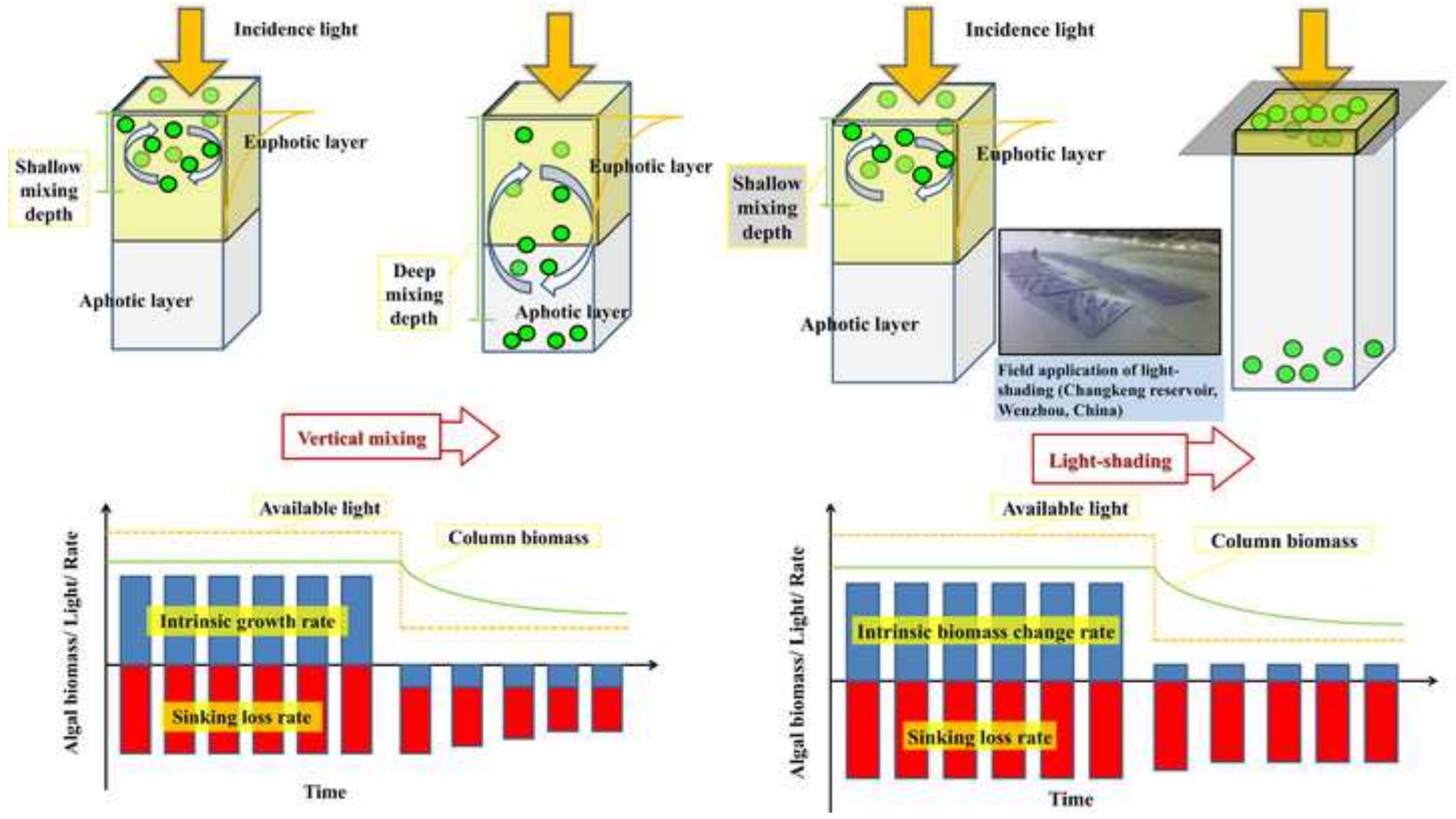